\section{www (WeltWeite Werbung') und die Folgen}

\section{Radikalisierung, Spionage, Vertrauens- und Wahrheitsverlust}

\author{
M. Spitzer, UIm
}

Die meisten von uns erleben Werbung so ähnlich wie Stubenfliegen im Sommer: Störend, meist nicht weiter beachtet, aber zuweilen unsere Aufmerksamkeit erregend, weil wir die Störung beseitigen wollen. Das geht jeweils sehr schnell - mit Fliegenklatsche einerseits oder Fernbedienung bzw. Mausklick andererseits. Lästig wird das Ganze, wenn man es im Sommerurlaub an einem stehenden Gewässer mit ganzen Armeen von Insekten und während der restlichen 50 Wochen des Jahres täglich mit mehreren Tausend „Ads“ - kleinen Text-, Bild- und Videoschnipseln - zu tun hat. So viele treffen tatsächlich auf unsere Augen und Ohren, wenn auch nur ein kleiner Teil davon (ca. 5-15\%) unsere bewusste Aufmerksamkeit erreicht und noch deutlich weniger „hängen bleibt“.

Selten machen wir uns klar, dass Werbung mit einem globalen Jahresumsatz von mehreren hundert Milliarden US Dollar zu den größten Wirtschaftszweigen überhaupt gehört. Noch seltener bedenken wir, dass die Umsätze bei Print- und Fernsehwerbung seit Jahren abnehmen (um Prozentsätze im einstelligen Bereich), wohingegen sie bei digitalen Medien jährlich steigen

1 Ich verdanke dieses Wortspiel mit der Abkürzung des World Wide Web dem Autor Marc-Uwe Kling, dem mit seiner Realsatire QualityLand ein großer Wurf gelungen ist. Dieser Artikel zeigt sehr deutlich, dass die Realität nicht nur Huxley und Orwell längst eingeholt hat, sondern sogar dieses geniale Kunstwerk, obwohl es noch nicht einmal ein Jahr alt ist. Ich empfehle jedem, der es noch nicht kennt die Lektüre wärmstens; besser noch: das Hörbuch!

\section{Nervenheilkunde 2018; 37: 303-311}

Korrespondenzadresse

Prof. Dr. Dr. Manfred Spitzer, Universitätsklinikum Ulm Klinik für Psychiatrie und Psychotherapie III

Leimgrubenweg 12, $89075 \mathrm{Ulm}$ (zweistellig), insbesondere in den Bereichen Unterhaltung (YouTube, OnlineStreaming-Dienste) und Social Media (Twitter, Facebook). Soweit die digitale Software. Hardwareseitig kommt die digitale Werbung heute vor allem über das Smartphone, dem „Schweizer Taschenmesser des Informationszeitalters“: Immer dabei, immer verfügbar und dauernd benutzt. Mittlerweile werden über 50\% des Werbeumsatzes mit dem Smartphone generiert und die Werbewirtschaft wächst und wächst. Dies mag Ökonomen gefallen - in der Medizin hingegen wird ungebremstes ausuferndes Wachstum als Krebs bezeichnet.

Mit 1,5 Milliarden Nutzern hat das Internet-Video-Portal YouTube das Fernsehen als Leitmedium längst abgelöst, gibt es doch deutlich weniger als 1,5 Milliarden Haushalte mit Fernsehapparat. Während wir jedoch früher den Fernseher einschalteten, um zu schauen, was wir wollten, werden heute etwa drei Viertel aller gesehenen YouTube-Videos von YouTube ausgewählt und vorgeschlagen. Hierfür verwendet YouTube einen Algorithmus, der von dessen Ingenieuren selbst als „eines der größten und raffiniertesten existierenden industriellen Systeme zur Empfehlung von Inhalten"2 bezeichnet wurde (9). Die Nutzung von YouTube liegt derzeit bei mehr als einer Milliarde Stunden täglich (19), was letztlich an der nie dagewesenen Kombination von Hardware (Smartphone) und Software (Auswahl durch YouTube) liegt.

\section{YouTube hat mit 1,5 Milliarden}

Nutzern und täglich einer Milliarde

Stunden Nutzung das Fernsehen als Leitmedium längst abgelöst.

Vordergründig sollen dem Verbraucher ähnliche Videos gezeigt werden wie dasje- nige, das er sich zunächst selbst ausgesucht hatte. In Wahrheit hat das System jedoch das Ziel, den Nutzer solange wie irgend möglich an die Mattscheibe des Computers, Tablets oder Smartphones zu binden, denn YouTube gehört Google, und Google lebt von Werbeeinnahmen. Je mehr Zeit jemand vor dem Bildschirm verbringt, desto mehr Werbung kann vor oder während der Video-Clips dargeboten werden und desto größer der Gewinn von Google. Wie andere großen Internetfirmen (Apple, Facebook, Twitter) lebt auch Google davon, die Aufmerksamkeit und damit die Zeit der Nutzer an zahlende Kunden zu verkaufen das ist schließlich das Geschäftsmodell aller Werbefirmen.

Schon im vergangenen Jahr war aufmerksamen YouTube-Nutzern aufgefallen, dass dort nach dem ersten, selbst ausgesuchten Video andere Videos empfohlen wurden (bzw. gleich anschließend einfach abgespielt werden; die Funktion heißt „autoplay"), die radikaler sind als das erste. Man beginnt bei „Joggen“ und landet wenige Videos später bei „Ultramarathon“; oder man beginnt mit „vegetarisch“ und trifft sehr bald auf „vegan“. Insbesondere bei politischen Inhalten wurde die Tendenz zur Radikalisierung sehr deutlich: Man startete (in den USA) mit Donald Trump und bekam sehr bald Videos von Leugnern des Holocaust oder Vertretern der Höherwertigkeit der weißen Rasse. Begann die YouTube-Video-Session mit Hillary Clinton, landete man bald bei linken Verschwörungstheorien, wie beispielsweise der, dass der US-amerikanische Staat selbst die Anschläge am 11. September 2001 ausgeführt habe (31).

\footnotetext{
2 Ausnahmsweise sei hier die gesamte Zusammenfassung der Arbeit im Original wiedergegeben, aus der das Zitat (vom Autor übersetzt) entnommen ist: "Abstract: YouTube represents one of the largest scale and most sophisticated industrial recommendation systems in existence. In this paper, we describe the system at a high level and focus on the dramatic performance improvements brought by deep learning. The paper is split according to the classic two-stage information retrieval dichotomy: first, we detail a deep candidate generation model and then describe a separate deep ranking model. We also provide practical lessons and insights derived from designing, iterating and maintaining a massive recommendation system with enormous user- facing impact."
} 


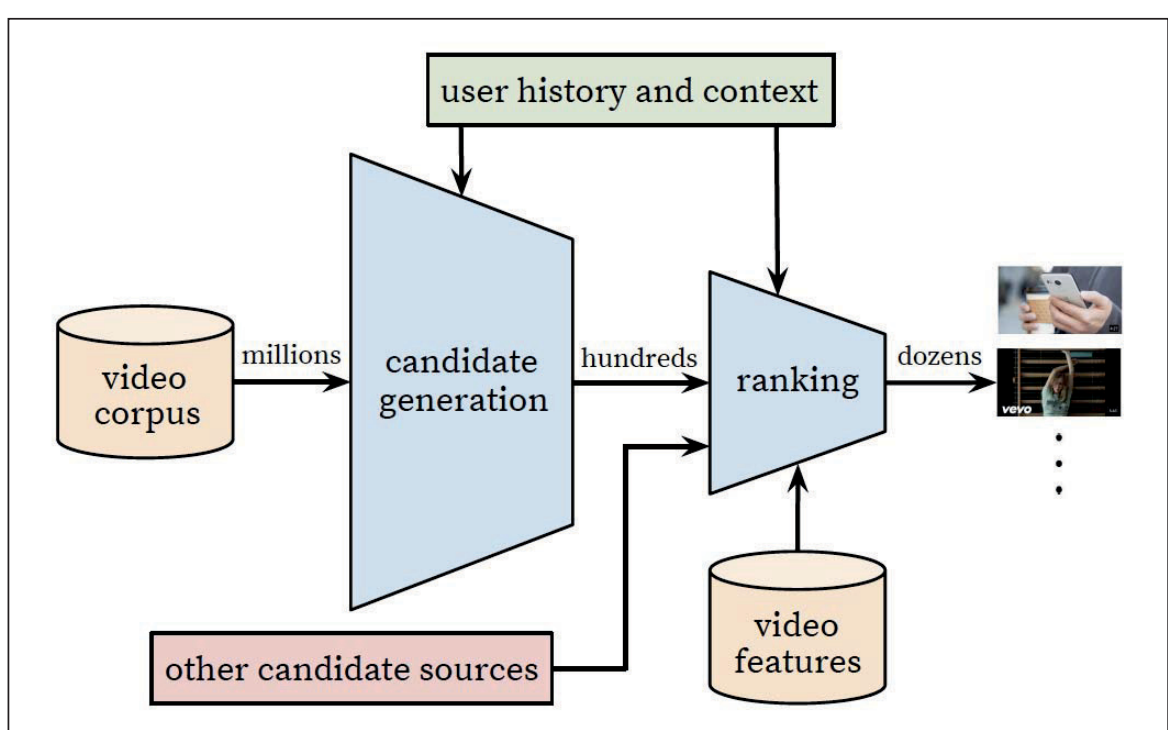

Abb. 1 Prinzip der trichterförmigen Architektur des neuronalen Netzwerks zur Ausführung der Empfehlungen für weitere Videoangebote an einen Nutzer (Figure 2 aus 9, S. 2). Aus Millionen Videos werden einige Hundert ausgewählt, die dann bewertet in eine Reihenfolge gebracht werden. Nur wenige Videos werden schließlich empfohlen bzw. automatisch gezeigt. Im Text der Arbeit heißt es hierzu: „Unsere Modelle lernen etwa eine Milliarde Parameter und werden anhand von Hunderten von Milliarden Beispielen trainiert" (S. 1; Übersetzung durch den Autor). ${ }^{3}$

Damit sehen sich weltweit 1,5 Milliarden Menschen für mehr als eine Milliarde Stunden Videos an, deren Inhalte automatisch radikaler sind als die Ansichten der Betrachter dieser Videos. Die Ursache dafür ist das Profitstreben der Werbeindustrie. Mittlerweile ist der Bewertungsalgorithmus nicht mehr von Menschen konstruiert, sondern Produkt von clever durchgeführtem machine-learning: Zwei neuronale Netzwerke arbeiten zusammen ( $\downarrow$ Abb. 1). Das erste generiert mögliche Kandidaten zur Empfehlung anhand von Eigenschaften der Videos und Ähnlichkeiten (die wiederum zumindest zum Teil aus dem Verhalten der Nutzer generiert werden). Das zweite bewertet die Kandidaten seinerseits aufgrund gelernter Bewertungen durch viele Nutzer und bringt sie damit in eine Rangreihenfolge (ranking). Dieser Ansatz erwies sich als besser geeignet zur Vorhersage der Zeit, die ein YouTuber vor dem Bildschirm verbringt (9) als frühere Modelle, die auf Algorithmen basierten.

\footnotetext{
3 Im Original: „Our models learn approximately one billion parameters and are trained on hundreds of billions of examples."
}

Die lernende Maschine tut nichts anderes als die Zeit vor dem Bildschirm zu maximieren. Sie hat weder "böse Absichten“ noch „will“ sie radikalisieren. Da jedoch radikalere, extremere Inhalte die Leute länger am Bildschirm kleben lassen, wählt die lernende Maschine automatisch solche Inhalte aus und empfiehlt sie. Die Radikalisierung ist also nicht intendiert, sondern vielmehr eine nicht intendierte Konsequenz der Zielfunktion (Maximierung der Zeit jedes einzelnen Betrachters vor dem Bildschirm) und der automatisierten Lernprozedur. Seit dem Jahr 2016 ist YouTube mit Hilfe dieser Technik wesentlich erfolgreicher darin, die Leute vor dem Bildschirm zu halten. Seit dem Jahr 2012 verzehnfachte sich die mit YouTube weltweit verbrachte Zeit.

Weil in neuronalen Netzwerken das "Wissen" in Milliarden von Verbindungen (den "Synapsen“) gespeichert ist, weiß niemand, wie sie funktionieren ${ }^{4}$. Man kann

4 Hierzu sei der „YouTube recommendations“ Ingenieur Jim McFadden zitiert: „Using a deep neural network makes the recommendations more of a black box to engineers than previous techniques [...] We don't have to think as much. [...] We'll just give it some raw data and let it figure it out." grundsätzlich nur feststellen, dass sie funktionieren, in manchen Fällen sogar schon besser als menschliche Gehirne. Diese sind in ihrer Lernfähigkeit - nicht zuletzt durch ihre Größe (ca. 100 Milliarden Nervenzellen mit einer Million Milliarden Verbindungen) und die Lebenszeit eines Menschen - begrenzt. Maschinen mögen - zurzeit noch - weniger leistungsfähig sein als Gehirne, auf längere Sicht jedoch wird sich dies ändern. Und so werden wir damit leben lernen müssen, dass uns Maschinen Empfehlungen geben, die wir nicht nachvollziehen können, aber glauben müssen etwa wie man im Altertum einem Orakel geglaubt hat, wie bereits vor etwa zwei Jahren im Fachblatt Nature zu lesen war (2).

Bekannt wurde die Radikalisierung als Funktionsprinzip von YouTube aufgrund einer Untersuchung, die das Wall Street Journal bei einem ehemaligen Mitarbeiter von YouTube, Guillaume Caslot, in Auftrag gegeben hatte (19). Dieser hatte bis 2013 bei YouTube am Empfehlungsalgorithmus gearbeitet und war dann - angeblich wegen $\mathrm{zu}$ geringer Arbeitsleistung - entlassen worden. In Wahrheit hatte sich Caslot gegenüber der extrem fragwürdigen Ethik hinter den YouTube-Empfehlungen kritisch gezeigt und wurde deswegen entlassen (31). Die Untersuchung von Caslot bestätigte, dass YouTube tatsächlich Nutzern, die ganz normale („mainstream“) Videos betrachteten, zunehmend radikalere Videos empfiehlt: „Suchtest Du nach Informationen über die Grippeimpfung, wurden Dir Videos mit Verschwörungstheorien von Impfgegnern empfohlen", beschreibt die New York Times in einer zusammenfassenden Titelgeschichte vom 8 . März 2018 die Situation. Die Untersuchung zeigte insgesamt:

„[...] die Empfehlungen von YouTube führen die Nutzer zu Kanälen, die Verschwörungstheorien, einseitige parteiische Meinungen und irreführende Videos verbreiten, selbst dann, wenn die Nutzer keinerlei Interesse an solchen Inhalten haben. Wenn die Nutzer eine politische Tendenz zeigen im Hinblick darauf, was sie sich zum Ansehen auswählen, empfiehlt ihnen YouTube typischerweise Videos, die diesen Vorurteilen entsprechen - oft mit extremeren Meinungen" (19; Übersetzung durch den Autor). ${ }^{5}$ 
Die Schießerei an der Schule in Parkland, Florida, im Februar 2018, wird beispielsweise von Tausenden YouTube-Videos als „falsche Nachricht mit gestellten Fotos zur Eindämmung der Waffenlobby“ (19), d. h. als Verschwörungstheorie dargestellt. Ebenso die Schießerei an einer Schule in Newtown im Jahre 2012 und die Schießerei während eines Konzerts in Las Vegas im Herbst 2017. Gibt man "Papst“ auf YouTube ein, erscheinen sehr bald auf Sensation ausgerichtete, falsche Nachrichten, bald nach der Eingabe „Sonnenfinsternis" zeigt YouTube ein 3000-fach gesehenes Video, das behauptet, die Erde sei eine Scheibe.

\section{Der Motor der Radikalisierung durch YouTube ist letztlich unsere eigene Neugier.}

Der Motor der Radikalisierung durch YouTube ist letztlich unsere Neugier, wie die New York Times unmissverständlich darlegt: „Wir sind Zeuge der mathematischtechnischen Ausbeutung eines natürlichen menschlichen Bedürfnisses: dem Wunsch, hinter den Vorhang von etwas zu schauen, einen tieferen Einblick in etwas, das uns angeht, zu gewinnen. Immer wenn wir klicken und klicken, werden wir von einer aufregenden Sensation zur nächsten geführt und entdecken immer mehr Geheimnisse und immer tiefere Wahrheiten. YouTube führt uns hinunter in ein Labyrinth des Extremismus, während Google seine Gewinne aus der Werbung steigert (31, S. 15; Hervorhebung und Übersetzung durch den Autor). ${ }^{6}$

5 „The Journal investigation found YouTube's recommendations often lead users to channels that feature conspiracy theories, partisan viewpoints and misleading videos, even when those users haven't shown interest in such content. When users show a political bias in what they choose to view, YouTube typically recommends videos that echo those biases, often with more-extreme viewpoints."

6 Im englischen Original noch prägnanter: „What we are witnessing is the computational exploitation of a natural human desire: to look "behind the curtain," to dig deeper into something that engages us. As we click and click, we are carried along by the exciting sensation of uncovering more secrets and deeper truths. YouTube leads viewers down a rabbit hole of extremism, while Google racks up the ad sales."
Der Autor vergleicht diese natürliche menschliche Neugier, die durch die Gegebenheiten des modernen Lebens gegen uns Menschen pervertiert wird, mit anderen menschlichen „Schwächen“, die uns ebenfalls erst in jüngerer Zeit schaden: Salz, Zucker und Fett. Früher waren diese Nahrungsbestandteile selten und wir suchten danach, um gesund und fit zu bleiben. Sind sie jedoch im Überfluss vorhanden und werden sie zudem permanent beworben, führt dies zu Übergewicht, Bluthochdruck und Diabetes und damit zum vorzeitigen Tod einer sehr großen Zahl von Menschen (27). In gleicher Weise führt uns unsere (eigentlich sehr gesunde) Neugier zu Lügen, Skandalen und Fehlinformation.

Mit den Worten des Autors: „Im Endeffekt hat YouTube ein Restaurant erschaffen, das uns immer süßere und fetthaltigere Speisen serviert und unsere Teller sofort nachfüllt, wenn wir mit einer Mahlzeit fertig sind. Mit der Zeit passt sich unser Geschmack an und wir bestellen daher Speisen, die immer mehr Zucker und Fett enthalten, die uns das Restaurant dann auch prompt serviert. Als daraufhin das Gesundheitsministerium und besorgte Bürger das Restaurant damit konfrontieren, antworten dessen Manager, dass sie doch nur das servieren, was die Leute wünschen und bestellen“ (31, S. 15; Übersetzung durch den Autor). ${ }^{7}$

Man kann ihm nur beipflichten wenn er anschließend betont, dass diese Situation aufgrund der vielen Nutzer von YouTube und vor allem der vielen jungen Nutzer ${ }^{8}$ von YouTube - besonders gefährlich ist. Bedenkt man noch, dass das preiswerte Chromebook von Google bei den Laptops für Schüler in den USA einen Marktanteil von über $50 \%$ hat und mit vorinstalliertem

\footnotetext{
7 Im Original noch schöner: „In effect, YouTube has created a restaurant that serves us increasingly sugary, fatty foods, loading up our plates as soon as we are finished with the last meal. Over time, our tastes adjust, and we seek even more sugary, fatty foods, which the restaurant dutifully provides. When confronted about this by the health department and concerned citizens, the restaurant managers reply that they are merely serving us what we want."

8 Nicht nur in Qatar (28) dürfte YouTube zu den häufigsten schon von Kindern im Kindergartenund Grundschulalter genutzten Anwendungen auf ihren Smartphones und Tablets gehören.
}

YouTube-Zugang geliefert wird, wird die Tragweite der automatisch durch YouTubeNutzung bewirkten Radikalisierung erst so richtig deutlich. Lassen wir den Autor daher ein letztes $\mathrm{Mal}$ in seiner Sprache $\mathrm{zu}$ Wort kommen: „This state of affairs is unacceptable but not inevitable. There is no reason to let a company make so much money while potentially helping to radicalize billions of people, reaping the financial benefits while asking society to bear so many of the costs. ${ }^{\text {"9 }}$ Recht hat er!

Wäre YouTube (und damit Google) die einzige zu fürchtende, große und superreiche US-amerikanische Firma, dann wäre dieser Artikel hier zu Ende. Leider ist dem nicht so. Betrachten wir als weiteres Beispiel Facebook, das mit über zwei Milliarden Nutzern größte soziale Online-Medium. Wie YouTube ist Werbung seine Geschäftsgrundlage. Um immer besser personalisierte Werbung an Nutzer zu versenden, sammelt Facebook die Daten seiner Nutzer und analysiert diese auf immer raffiniertere Weise - wie Google auch unter Zuhilfenahme neuester machine-learning Verfahren.

\section{Facebook weiß über einen bestimmten Menschen ebenso gut Bescheid wie dessen Ehepartner.}

Welche Daten? - Früher mussten Werbefirmen die Vorlieben der Nutzer per Fragebogen erfassen, dann mit bestimmten erfragten Variablen (z. B. Alter, Geschlecht, Beruf) verknüpfen, dann noch bestimmte Eigenschaften der Nutzer ebenfalls erfragen, um beispielsweise herauszufinden, dass große Frauen eher Turnschuhe tragen. Das war sehr aufwändig! Heute braucht man nichts weiter zu tun als die Clicks (auf z. B. überall auf dem Bildschirm aufflackernde Anzeigenwerbung) und Likes (in Facebook) oder den Twitter-Account einer Person $\mathrm{zu}$ analysieren und schon kennt man deren Alter, Geschlecht, Intelligenz, sexuelle Präferenz, Beziehungsstatus, poli-

9 „Diese Lage der Dinge ist nicht akzeptierbar, aber nicht unvermeidbar. Es gibt keinen Grund dafür, eine Firma so viel Geld verdienen zu lassen, indem sie potenziell dazu verhilft, Milliarden von Menschen zu radikalisieren, und gleichzeitig der Gesellschaft die Kosten hierfür aufdrückt.“ 


\section{Today is Election Day}

Find your polling place on the U.S.

$\begin{array}{ll}\star \star \star & \text { Politics Page and click the "I Voted" } \\ \text { VOTE } & \text { button to tell your friends you voted. } \\ \star \star \star & \text { I Voted }\end{array}$

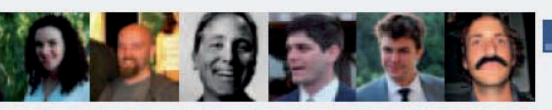

Jaime Settle, Jason Jones, and 18 other friends have voted.

What's this? • close

\section{\begin{tabular}{l|l|l|l|l|l|l|l}
0 & 1 & 1 & 5 & 5 & 3 & 7 & 6
\end{tabular}}

People on Facebook Voted son

\section{Today is Election Day

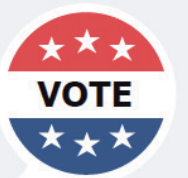 \\ Find your polling place on the U.S. Politics Page and click the "I Voted" button to tell your friends you voted. \\ I Voted What's this? - close}

Abb. 2 Beispiele der Facebook-Nachrichten, die an 60055176 Nutzer der Experimentalgruppe „soziale Nachricht" (d. h. Nachricht mit Bildern), an 611044 Nutzer der Experimentalgruppe "(rein) informationelle Nachricht" (d. h. Nachricht ohne Bilder) und an 613096 Nutzer der Kontrollgruppe (keine Nachricht) gesendet wurden.

tische und religiöse Weltanschauung sowie eine ganze Reihe weiterer persönlicher Daten wie Haltungen, Vorlieben, Werte, Einstellungen, Interessen, Ziele und Wünsche.

Dass dies geht und wie gut es im Einzelfall geht, wurde bereits vor fünf Jahren im Fachblatt PNAS publiziert (13; für eine Übersicht, vgl. 26). Mittlerweile weiß man sogar, wie gut sich aus den "Likes“ eines Facebook-Nutzers dessen Persönlichkeitseigenschaften („Big Five“), politischer Hintergrund, sexuelle Orientierung und vieles mehr ableiten lassen. Mit nur 9 FacebookLikes kann man die Persönlichkeit eines Menschen etwa so gut vorhersagen wie das einer von dessen Arbeitskollegen kann, mit 65 Likes ist man so gut wie ein Freund, mit 125 Likes so gut wie Vater, Mutter, Bruder oder Schwester (34). Mit den 225 Likes, die Facebook-Nutzer im Durchschnitt abgegeben haben, ist jeder, der diese Daten auswerten kann, so gut wie der Partner! Kurz: Internetfirmen wie Facebook wissen so gut wie der (Ehe-)Partner, wer ein bestimmter Mensch ist, wie er lebt und was ihn umtreibt!
Man kann sogar dessen Gedanken, Gefühle und Verhalten beeinflussen. Im Januar 2012 wurden knapp 700000 Nutzern von Facebook eine Woche lang manipulierte Startseiten mit veränderten Statusmeldungen ihrer Freunde angezeigt. Mitarbeiter von Facebook wollten untersuchen (bei fragwürdiger Ethik der ganzen Studie, 32), ob und wie die Manipulation von Emotionen von Hunderttausenden von FacebookNutzern durch die Art, wie Nachrichten dargeboten werden, gelingen kann. Per Zufallsauswahl (Randomisierung) bekam ein Teil der Nutzer vor allem positive Statusmeldungen zu sehen, der andere Teil dagegen überwiegend negative. Ausgewertet wurden dann die Auswirkungen dieser experimentellen Manipulation der Emotionen der Nutzer auf deren Posting-Verhalten (15). Man fand tatsächlich eine Änderung von deren Emotionen in die jeweils manipulierte Richtung.

Facebook kann Gedanken, Gefühle und Verhalten manipulieren.
Eine im Fachblatt Nature bereits im Jahr 2012 publizierte, kontrollierte, randomisierte Studie an über 61 Millionen Facebook-Nutzern hatte zudem ergeben, dass das Wählerverhalten bei den Kongresswahlen im Jahr 2010 durch entsprechende Verlautbarungen in Facebook - sogenannte „Posts" - messbar beeinflusst wurde (6, S. 295): „Die Ergebnisse zeigen, dass die Nachrichten den Ausdruck der politischen Meinung, die Informationssuche und das Wahlverhalten von Millionen von Leuten direkt beeinflussen. Darüber hinaus beeinflussen die Nachrichten nicht nur die Nutzer, an die sie gerichtet waren, sondern auch die Freunde der Nutzer und wiederum deren Freunde ${ }^{\prime 10}$, schreiben die Autoren in ihrer Zusammenfassung.

Sie hatten in einem sehr cleveren Versuchsdesign die Auswirkung der Facebook-Nachricht „ich habe gewählt" untersucht - entweder mit den Bildern einiger Facebook-Freunde oder ohne diese. Eine weitere (Kontroll-)Gruppe bekam gar keine Nachricht ( $\triangleright$ Abb. 2).

Die relative Häufigkeit der Klicks auf „I voted" wurde als Maß für das Bedürfnis der Leute verwendet, sich selbst als Wähler zu identifizieren. Dies waren in der Gruppe „soziale Nachricht" 20,04\% und damit 2,08\% mehr als in der Gruppe ,informationelle Nachricht" mit 17,96\% (SEM = $0,05 \%, p<0,01)$. Dies bedeutet, dass das Betrachten der Gesichter von Freunden einen deutlichen Effekt auf das Verhalten der Wähler hatte. Mit weiteren Verfahren fanden die Autoren, dass sich das Anklicken von „I voted“ auch auf die Informationssuche (nach dem Wahllokal; durch Klicken des Schaltfläche „find your polling place...") und das tatsächliche Wählen (mit $0,39 \%$; SEM $=0,17 \%, p=0,02$ ) auswirkten. ${ }^{11}$

10 „The results show that the messages directly influenced political self-expression, information seeking and real-world voting behavior of millions of people. Furthermore, the messages not only influenced the users who received them but also the users' friends, and friends of friends."

11 Die Autoren sprechen hier von validiertem Wählen und verweisen auf das ziemlich ausführliche Supplement für genauere Angaben dazu, wie dies im Einzelnen erfasst wurde. „[...] we also measured the effect that the experimental treatment had on validated voting, through examination of public voting records" (6, S. 295). 
Nun könnte man einwenden, dass die Stärke des Effekts mit knapp 0,4\% nun wirklich nicht berauschend sei und nur aufgrund der enormen Zahl der untersuchten Teilnehmer $(n=61279316)$ statistisch signifikant wurde. Dem ist jedoch nicht so, denn sogar sehr kleine Effekte können eine große Wirkung haben, wie der Statistiker und Psychologe Robert (Bob) Rosenthal schon vor Jahrzehnten für die Bereiche Psychologie und Medizin hervorhob. Bei den US-Präsidentschaftswahlen ist das noch eindrücklicher, wie das folgende Beispiel zeigt:

Ich kann mich noch gut an die US-Präsidentschaftswahlen im Jahr 2000 erinnern, nicht zuletzt deswegen, weil ich sie zusammen mit Freunden während des Jahreskongresses der Society of Neuroscience in New Orleans (vor Hurrikan Katrina!) miterlebte. Damals - der Demokrat Clinton hatte seine 2. Amtszeit hinter sich - war der Demokrat Al Gore der Favorit gegenüber dem Republikaner George W. Bush. Gore bekam auch tatsächlich gut eine halbe Million mehr Stimmen (50994086) als Bush (50 461 092), dessen Stimmen jedoch besser auf die Wahlmänner der einzelnen Bundesstaaten verteilt waren. Letztlich siegte Bush, weil er in Florida, dem für den Ausgang der Wahl entscheidenden Bundesstaat, eine Mehrheit von ganzen 538 Wahlmännerstimmen für sich gewinnen konnte. Knapper wurde in den USA noch nie eine Wahl gewonnen. Bedenkt man zudem, dass es einen dritten Kandidaten gab (den „Grünen“ Ralph Nader), dessen 2882728 Stimmen mehrheitlich Al Gore zu Gute gekommen wären (hätte Nader auf seine ohnehin aussichtslose Kandidatur verzichtet), dann sieht man

- wie bitter die Niederlage von Al Gore wirklich war (die Mehrheit wollte seine Politik, nicht die von Bush),

- wie groß die Auswirkungen eines winzigen Effekts (537 Stimmen waren weniger als $0,01 \%$ der in Florida abgegebenen Stimmen) und

- wie anfällig letztlich jedes demokratische Wahlsystem ${ }^{12}$ für Manipulationen sein kann, solange deren Effekt nicht Null ist!

Rechnet man die von Bond und Mitarbeitern gewonnenen Ergebnisse auf die US-
Tab. 1

Inhalte von gekauften Facebook-Werbeanzeigen (Beispiele aus der Tabelle auf S. 20 der Anklageschrift, überschrieben mit "The political advertisements included the following")

\begin{tabular}{l|l}
\hline $\begin{array}{l}\text { Approximate } \\
\text { Date }\end{array}$ & Excerpt of Advertisement \\
\hline April 6, 2016 & $\begin{array}{l}\text { "You know, a great number of black people support } \\
\text { us saying that \#HillaryClintonlsNotMyPresident" }\end{array}$ \\
\hline April 7, 2016 & "I say no to Hillary Clinton / I say no to manipulation" \\
\hline April 19, 2016 & "JOIN our \#HillaryClintonForPrison2016" \\
\hline May 10, 2016 & $\begin{array}{l}\text { "Donald wants to defeat terrorism ... Hillary wants } \\
\text { to sponsor it" }\end{array}$ \\
\hline May 24, 2016 & "Hillary Clinton Doesn't Deserve the Black Vote" \\
\hline June 7, 2016 & "Trump is our only hope for a better future!" \\
\hline June 30, 2016 & $\begin{array}{l}\text { "\#NeverHillary \#HillaryForPrison \#Hillary4Prison } \\
\text { \#Trump \#Trump4President" }\end{array}$ \\
\hline July 20, 2016 & "Ohio Wants Hillary 4 Prison" \\
\hline August 10, 2016 & $\begin{array}{l}\text { "We cannot trust Hillary to take care of our vete- } \\
\text { rans!" }\end{array}$ \\
\hline October 19, 2016 & "Hillary is a Satan, and her crimes and lies had pro- \\
ved just how evil she is."
\end{tabular}

Bevölkerung hoch, dann ergibt sich (wir reden von den Kongresswahlen im Jahr 2010!), dass Facebook-Freunde $60000 \mathrm{zu}$ sätzliche Stimmen direkt und nochmals 280000 Stimmen indirekt (über die Freunde von Freunden) bewirkten. Diese 340000 Stimmen entsprechen $0,14 \%$ der gesamten Wahlpopulation.

\section{Wahlen in den USA und in Europa wurden durch Facebook beein- flusst.}

Einem online veröffentlichten Kommentar des Senior-Autors zufolge, wurden ähnliche Ergebnisse der Beeinflussbarkeit auch für die Präsidentschaftswahlen im Jahr 2012 gefunden: „A previous large scale experiment showed that a single message posted on social media could directly influence real world voting behavior, and that the indirect effect of the message on friends accounted for most of its total effect on increased voter turnout. Here, we analyze a follow-up experiment conducted in the

12 Übrigens: Es gibt sehr schöne Arbeiten darüber, dass demokratische Wahlsysteme, ganz gleich welches, nie zu 100\% den Wählerwillen abbilden, sondern immer „praktikable Kompromisse“ darstellen. Wie auch immer man es konstruiert, jedes Wahlsystem kann im Extremfall genau den Kandidaten als Gewinner hervorbringen, den nur eine Minderheit möchte.
2012 US Presidential Election. The results show that messaging had both direct and indirect effects on voting behavior in that election as well, suggesting that social media can be an effective tool for mobilizing political participation in high stakes elections. (James H. Fowler Professor University of California, San Diego; Hervorhebung durch den Autor).

Diese Erkenntnisse verblieben leider nicht im Bereich der Wissenschaft, sondern wurden ganz „praktisch“ umgesetzt, um damit Geld zu verdienen - im Rahmen der letzten Präsidentschaftswahlen in den USA. Seit der genannten Studie von Kramer und Mitarbeitern wusste man ja bereits, dass die Manipulationen von Menschen in großem Stil mit Facebook möglich ist.

Die Enthüllungen hierzu haben in der Woche vom 18. bis 25. März 2018 zum Wertverlust von Facebook an der Börse von 75 Milliarden US Dollar geführt ${ }^{13}$ und lassen sich beim heutigen Stand des Wissens (1. April 2018) in aller Kürze wie folgt zusammenfassen:

Bereits im Juli 2016 berichtete die Zeitschrift The New Yorker (8) erstmals über sogenannte russische Troll-Farmen bzw. Troll-Armeen zur Beeinflussung des USPräsidentschaftswahlkampfs im Herbst 2016. ${ }^{14}$ Die Recherchen des hierzu seit 17. Mai 2017 tätigen Sonderermittlers Robert 
S. Mueller haben mittlerweile zur Anklage von 13 russischen Staatsbürgern (unter ihnen der Milliardär und Putin-Vertraute Yevgeny Prigozhin) sowie russischer Internetfirmen (u. a. einer Troll-Farm, die den Namen Internet Research Agency trägt und Prigozhin gehört) geführt (30). Die Anklagepunkte lauteten Verschwörung gegen die Vereinigten Staaten, Betrug im Überweisungsverkehr, Bankbetrug und Identitätsdiebstahl. Den Angeklagten wird vorgeworfen, über Facebook und andere soziale Online-Medien rechtswidrigen Einfluss auf die Präsidentschaftswahlen im Jahr 2016 genommen $\mathrm{zu}$ haben, um dem heutigen Amtsinhaber Donald Trump einen Vorteil gegenüber seiner Konkurrentin Hillary Clinton zu verschaffen. In der im Internet frei zugänglichen Anklageschrift kann man hierzu lesen:

„By approximately May 2014, Defendants and their co-conspirators discussed efforts to interfere in the 2016 U.S. presidential election. Defendants and their coconspirators began to monitor U.S. social media accounts and other sources of infor-

13 Nach einer vom Meinungsforschungsinstitut Emnid für das Magazin Focus durchgeführten Umfrage (4) hat aus Sorge um den Datenschutz fast jeder zweite Nutzer von sozialen Medien in Deutschland bereits über eine Abmeldung nachgedacht. Männer (53\%) tendierten eher zu einer Abmeldung bei Facebook, Instagram oder Twitter als Frauen (44\%). Auch in den USA ist die Zukunft von Facebook ungewiss. Der Analyst Brian Wieser von der in Portland, OR, ansässigen Finanz-Beratungsfirma Pivotal Research Group schrieb in der Zeitschrift The Economic Times am 22.3.2018, dass Facebook möglicherweise zu groß geworden sei, wodurch es zu Missmanagement gekommen sei. „Investors now have to consider whether or not the company will conclude that it has grown in a manner that has proven to be untenable“ (6).

14 Die meisten von uns werden sich noch gut an die Zeit vor den US-Wahlen erinnern, während der immer wieder von den jahrzehntelangen Geschäftsbeziehungen von Trump mit Russland die Rede war und in denen Trump und Putin sich gegenseitig lobten, gemeinsame Positionen (u. a. Kritik an der NATO) vertraten und man den Eindruck gewinnen konnte, sie sprachen sich ab. Hierzu Chen: „[...] given the sheer volume and often menacing tenor of the coverage of the Trump-Putin connection, the casual reader would be forgiven for coming away with the strong suspicion that the two meet every month for strategy sessions over caviar at Putin's Black Sea dacha. 'There's something very strange and disturbing going on here, and it should not be ignored," Paul Krugman [Ökonomie-Nobelpreisträger] warned, in the Times." mation about the 2016 U.S. presidential election. By 2016, Defendants and their coconspirators used their fictitious online personas to interfere with the 2016 U.S. presidential election. They engaged in operations primarily intended to communicate derogatory information about Hillary Clinton, to denigrate other candidates such as Ted Cruz and Marco Rubio, and to support Bernie Sanders and then-candidate Donald Trump“ (1, S. 16f).

Die in der Anklageschrift genannten russischen Bürger hatten in großem Stil Facebook-Werbeanzeigen zur Denunzierung von Donald Trumps Gegenkandidatin Hillary Clinton gekauft ( $>$ Tab. 1). Aus dem Wortlaut der Anklageschrift (1, S. 19) wird deutlich, dass diese Aktivitäten eindeutig illegal waren. Das Unternehmen Facebook hat also durch kriminelle Handlungen Geld verdient: ${ }^{15}$ „From at least April 2016 through November 2016, Defendants and their co-conspirators, while concealing their Russian identities and ORGANIZATION [gemeint ist die russische Firma Internet Research Agency] affiliation through false personas, began to produce, purchase, and post advertisements on U.S. social media and other online sites expressly advocating for the election of then-candidate Trump or expressly opposing Clinton. Defendants and their co-conspirators did not report their expenditures to the Federal Election Commission, or register as foreign agents with the U.S. Department of Justice."

Mittlerweile wurden hochrangige Mitarbeiter des Wahlkampfteams von US-Präsident Donald Trump angeklagt und zum Teil verurteilt, was durch ebenfalls öffentlich zugängliche Gerichtsakten belegt ist. Im Zusammenhang der Ermittlungen wurde bekannt, dass sich das britische Unternehmen Cambridge Analytica bereits im Jahr 2014 die Daten von 87 Millionen Facebook-Nutzern verschafft hatte (22). Der damalige Mitarbeiter Aleksandr Kogan hatte eine Facebook-App programmiert, mit der Nutzer einen Persönlichkeitstest bei sich durchführen können. Dies taten

15 Daran ändern die Beteuerungen des FacebookGründers und -Chefs Mark Zuckerberg, dass er sich künftig mehr um die Wahrheit und die Datensicherheit kümmern werde (5), gar nichts.
270000 Leute und ahnten nicht, dass dabei nicht nur ihre eigenen persönlichen Daten, sondern auch die Daten all ihrer Freunde insgesamt eben 87 Millionen $(5,35)$ - erfasst wurden.

Dass Facebook letztlich die gleiche Aufgabe hat wie YouTube mit seinem Empfehlungsalgorithmus, hat der Gründungspräsident der Firma, der Milliardär (und zudem Mitbegründer der Musiktauschbörse Napster) Sean Parker, unlängst selbst zugegeben: "Wie bekommen wir so viel wie möglich von Ihrer Zeit und bewussten Aufmerksamkeit?"16 (25) war dessen zu beantwortende grundlegende Frage. Facebooks Lösung wurde bereits diskutiert: Die Werbung wird auf jeden einzelnen der etwa 2 Milliarden Nutzer persönlich zugeschnitten. Nach einer im Herbst 2017 im Fachblatt PNAS publizierten Feldstudie an 3,5 Millionen Facebook Nutzern ist solche personalisierte, d. h. auf die Persönlichkeitseigenschaften und Interessen einzelner Nutzer zugeschnittene Werbung um etwa 50\% effektiver als übliche (nicht personalisierte oder falsch personalisierte) Werbung (18).

\section{Werbung, die auf die Persönlich- keitseigenschaften und Interessen einzelner Nutzer zugeschnitten ist, erwies sich als um etwa $50 \%$ effektiver als herkömmlich, nicht personalisierte Werbung.}

Die Firma Cambridge Analytica hatte nun als eine der ersten erkannt, dass man diese Art der Personalisierung nicht nur zur gezielteren und effektiveren Werbung, sondern auch zum Zweck der politischen Einflussnahme verwenden konnte (21). So wurden die in Tabelle 1 beispielhaft angeführten „Nachrichten“ gezielt von scheinbaren oder tatsächlichen US-Bürgern an „Gleichgesinnte" (identifiziert von Cambridge Analytica) geleitet, und zwar immer zum gerade ablaufenden Tagesgeschehen des Wahlkampfes passend: Nudging in sehr großem Stil also. Was dabei herauskam, ist (Welt-)Geschichte.

Wenn man sich vor Augen führt, dass Fake-News während der letzten US-Präsi-

16 "How do we consume as much of your time and conscious attention as possible?" 
dentenwahlen deutlich häufiger proTrump- als pro-Clinton-Inhalte betrafen (31, S. 15), wird deutlich, wie stark demokratische Prozesse durch digitale Informationstechnik gefährdet sind. Nimmt man hinzu, dass diese falschen Nachrichten von außen (Russland) gesteuert wurden, wird die Brisanz dieses Befundes noch deutlicher. Und es wäre schön, wenn dies alles nur für die Amerikaner gelten würde. Aber leider spielte sich bei unseren unmittelbaren europäischen Nachbarn mehrfach das Gleiche ab: So beobachtete man bei den letzten Präsidentschaftswahlen in Tschechien (am 27. Januar 2018) ein Kopf-anKopf-Rennen des „alten Kandidaten“ Miloš Zeman ${ }^{17}$, einem vermutlich alkoholkranken Populisten, mit dem Herausforderer Jiř́ Drahoš, einem Professor für physikalische Chemie und zugleich dem Leiter der Tschechischen Nationalen Akademie der Wissenschaften. Mit etwa 3\% Vorsprung gewann Zeman. Im Vorfeld hatte es massive Fake-News zu Lasten des neuen Kandidaten gegeben. Unglaublich viele negative Gerüchte wurden über ihn gestreut, dem Wahlvolk damit Angst gemacht und möglicherweise die Wahl dadurch zu dessen Ungunsten beeinflusst. Für die letzten Präsidentschaftswahlen in Polen gilt Ähnliches, für den Brexit im Vereinigten Königreich auch.

$\mathrm{Zu}$ den beschriebenen Mechanismen der automatischen Radikalisierung (YouTube bzw. Google) und der bewussten Einflussnahme auf Wahlen (Spionage einschließlich Hochverrat) gesellt sich eine weitere Eigenschaft sozialer Medien: Die automatische Verstärkung von Lügen, also ein Verlust an Wahrheit in der Gesellschaft. Denn Lügen verbreiten sich viel schneller als die Wahrheit, vor allem online. Dies ist das Ergebnis einer im Fachblatt Science von Wissenschaftlern am Massachusetts Institute of Technology (MIT) publizierten Studie. In der Studie wurde die etwa 4,5 Millionen-malige (Weiter-)Verbreitung von etwa 126000 wahren und falschen Geschichten auf der Kommunikationsplattform

17 Miloš Zeman ist seit dem 8. März 2013 der dritte Staatspräsident der Tschechischen Republik, nachdem er zwischen 1998 und 2002 bereits Ministerpräsident war.
Twitter $^{18}$ in den Jahren 2006 bis 2017 durch etwa drei Millionen Menschen untersucht (33). Mit weltweit mehr als dreihundert Millionen Nutzern gilt Twitter nach Facebook als eines der bedeutsamsten sozialen Netzwerke, in dem Privatpersonen, Organisationen, Unternehmen und Massenmedien kurze (max. 280 Zeichen) Textnachrichten (Tweets) im Internet verbreiten. Die Einteilung der Nachrichten als wahr oder falsch erfolgte mittels sechs unabhängiger Organisationen ${ }^{19}$, die Fakten-Checks durchführen und deren Klassifikationen zu $95 \%$ bis $98 \%$ übereinstimmten. Nach dem Inhalt wurden die Nachrichten zudem nach "Naturkatastrophen“, „Terrorismus“, „Wissenschaft", „(fiktionale) Anekdoten“ bzw. „Großstadtlegenden“ und „Wirtschaftsnachrichten" klassifiziert.

Methodisch ging man hierzu wie folgt vor: Wenn jemand ein „Gerücht" ${ }^{\text {"20 }}$ (irgendeine Behauptung über Twitter) verbreitet, dann kommt es vor, dass diese Behauptung durch andere Nutzer von Twitter weiterverbreitet wird, sodass eine Kaskade entsteht. Wird die Behauptung 10-mal weiterverbreitet, dann beträgt die Länge der Kaskade 10, wird sie gar nicht weiter verbreitet, ist die Länge der Kaskade gleich 1. Wird die gleiche Behauptung von zwei Nutzern verbreitet, entstehen zwei Kaskaden.

Falsche Nachrichten sind unwahrscheinlicher und interessanter als wahre, und verbreiten sich auf Twitter daher deutlich schneller.

Die Autoren untersuchten nun solche auf Twitter aufzufindenden Kaskaden und be-

18 Twitter wurde im März 2006 vom US-amerikanischen Softwareentwickler und Unternehmer Jack Dorsay $\left({ }^{*} 1976\right)$ zunächst unter dem Namen $t w t t r$ gegründet. Wörtlich übersetzt bedeutet das Wort „Gezwitscher“. Eine Kurznachricht wird Tweet genannt, das Verb „to twitter“ (neudeutsch: „twittern“) bedeutet wörtlich „zwitschern“. Das Besondere an der Internetplattform besteht u. a. darin, dass man „Follower“ (Nutzer, welche die Nachrichten bestimmter Personen oder Institutionen gleichsam „subscribiert“ haben) haben kann, durch die eigene Nachrichten dann weiter verbreitet werden. Man spricht von re-tweet

19 Daten der folgenden Fakten-Check-Organisationen wurden hierzu herangezogen: Snopes.com, politifact.com, factcheck.com, truthorfiction.com, hoaxslayer.com und urbanlegends.about.com.

20 Die Autoren sprechen von „rumor“. stimmten verschiedene Maße der Diffusion von Gerüchten: Die Tiefe der Kaskaden als Anzahl der Weiterleitungen („retweets") durch verschiedene individuelle Nutzer über die Zeit; deren Größe (Anzahl der in die Kaskade involvierten Nutzer über die Zeit), maximale Breite (Anzahl der Nutzer in gleicher Tiefe) sowie deren "Viralität", wobei es sich um ein komplexes Maß der Diffusion handelt.

Falsche Nachrichten verbreiten sich im Vergleich zu wahren Nachrichten schneller, weiter und tiefer als wahre Nachrichten, oder wie ein anonymes Editorial im New Scientist es formulierte, „fake news [...] can be halfway around the world before the truth has got its boots on" (3). Wahre Nachrichten brauchten im Vergleich zu falschen Nachrichten sechs Mal so lang bis sie 1500 Leute erreichten. Falsche Nachrichten wurden zudem mit $70 \%$ höherer Wahrscheinlichkeit mit anderen Nutzern geteilt als wahre. Die am häufigsten verbreiten falschen Nachrichten (Top 1 Prozent) wurden für gewöhnlich tausend bis hunderttausend Mal weiterverbreitet, wohingegen wahre Nachrichten nur selten mehr als tausend Mal weiterverbreitet wurden.

Woran liegt das? - Auch dieser Frage gingen die Autoren nach. Mittels Textanalyse-Software konnten sie zeigen, dass falsche Nachrichten einen größeren Neuigkeitswert hatten als richtige. Dies wundert nicht, würde doch die Nachricht „der Papst ist schwanger" uns alle in höchstem Maße überraschen, denn ein Mann kann nicht schwanger werden und vom Papst würde man dies von allen bekannten Männern am wenigsten vermuten. Falsche Nachrichten sind daher nahezu definitionsgemäß in höherem Maße überraschend als wahre Nachrichten. Hinzu kommt der Befund, dass falsche Nachrichten neben der Überraschung auch Ekel hervorrufen, also zwei sehr starke Emotionen. Wahre Nachrichten dagegen bewirken je nach dem Inhalt eher Trauer, Freude, Vertrauen oder deren Vorwegnahme, wobei es sich (worauf die $\mathrm{Au}$ toren nicht eingehen) nur bei den ersten beiden Reaktionen um Emotionen handelt, die anderen beiden hingegen Verhaltensdispositionen beschreiben.

Die Autoren konnten weiter durch Software (sie verwendeten zwei unterschiedliche Verfahren) Bots identifizieren, die pro- 
grammiert sind, bestimmte Nachrichten zu senden bzw. weiterzuleiten, von denen man vermuten könnte, dass sie ebenfalls falsche Nachrichten eher verbreiten. Dem ist jedoch nicht so. Zwar konnte gezeigt werden, dass ihre Anwesenheit die Verbreitung von Wahrheit und Falschheit beschleunigt, nicht jedoch bevorzugt die von Falschheit.

Die Wissenschaftler vom MIT kommen daher in dieser bislang größten und methodisch aufwändigsten Studie zur Verbreitung von Gerüchten zu dem Schluss, dass es an uns Menschen liegt, dass sich die Unwahrheit schneller und weiter verbreitet als die Wahrheit. Genauer gesagt liegt es an unseren Emotionen und unserem Hunger nach unerwarteten bzw. überraschenden Nachrichten. Dieser lässt sich mit Unwahrheiten viel leichter stillen als mit langweiligen Wahrheiten: „Der Papst ist schwanger“ ist viel interessanter als „der Papst ist nicht schwanger"!

\section{Wenn die Gehirne von Milliarden Menschen systematisch mit falschen und radikalen Inhalten versorgt werden, kann das eines nicht haben: keine Auswirkungen!}

Werbung ist das Geschäftsmodell einiger der größten IT-Firmen der Welt (Google/ YouTube, Facebook, Twitter), d. h. ein beträchtlicher Teil der Weltbevölkerung bezahlt mit seiner Zeit und Aufmerksamkeit vor dem Bildschirm. Wenn die Gehirne von Milliarden Menschen dadurch systematisch mit falschen und radikalen Inhalten versorgt werden, kann das eines nicht haben: keine Auswirkungen! Wenn dann auch noch kriminelle Energie hinzu kommt und unsere demokratischen Prozesse zur Profitmaximierung nachweislich beeinflusst werden (wie durch Facebook), wird es höchste Zeit, nicht nur nachzudenken, sondern auch zu handeln! Müssen wir die Geschäftsgrundlage der genannten Firmen wirklich mit Steuergeldern (für den Breitbandausbau) weiter öffentlich fördern? Kann eine Gesellschaft auf der Grundlage automatischer und systematischer Spionage, Unwahrheit und Radikalisierung überhaupt nachhaltig existieren? Oder schaufeln wir uns längst alle unser Grab - mit einem vom bunten Flachbildschirm gesteuerten Gehirn?
Wie eine kürzlich von spanischen Autoren publizierte Arbeit (7) erstmals zeigen konnte, ist der Facebook-Skandal noch deutlich größer als hierzulande in der Presse publiziert - wo es ja vor allem um die USA und die wahrscheinlich vor allem dort geklauten 50 Millionen privaten Nutzerdatensätze geht. Auch diesseits des Atlantiks wurden nämlich nachweislich etwa 40\% aller Bürger der Europäischen Union - etwa 200 Millionen! - von Facebook analysiert, um personalisierte Werbung mittels sehr sensibler Daten zu versenden. Aufgefallen war dies einem der spanischen Autoren, der plötzlich Werbung für ein Hotel, das speziell Homosexuelle anspricht, erhalten hatte. Nun war er jedoch nicht homosexuell und wunderte sich darüber, wieso Facebook ihm diese Werbung geschickt hatte. Wie die ziemlich aufwändig durchgeführten Recherchen der Gruppe ergaben, wurden von 73\% der europäischen FacebookNutzer (was etwa 40\% aller EU-Bürger entspricht) sensible Daten ${ }^{21}$ im Sinne der EUDatenschutz-Grundverordnung (EU General Data Protection Regulation - GDPR) durch Algorithmen ermittelt und zu Werbezwecken verwendet. Dies widerspricht der Verordnung, die bereits seit dem 24. Mai 2016 in Kraft getreten ist, weswegen Facebook in Spanien mittlerweile zu einer Zahlung von 1,2 Millionen Euro verurteilt wurde. Wenn diese Verordnung am 25. Mai 2018 in der gesamten EU endgültig gilt (bislang leben wir datenschutzrechtlich in einer Übergangszeit), könnte das bedeuten, dass Facebook von der EU mit bis zu 4\% des Jahresumsatzes bestraft werden kann.

Kann eine Gesellschaft auf der Grundlage automatischer und systematischer Spionage, Unwahrheit und Radikalisierung überhaupt nachhaltig existieren?

21 Sensible Daten im Sinne der GDPR sind „Daten, aus denen die rassische und ethnische Herkunft, politische Meinungen, religiöse oder weltanschauliche Überzeugungen oder die Gewerkschaftszugehörigkeit hervorgehen, sowie die Verarbeitung von genetischen Daten, biometrischen Daten zur eindeutigen Identifizierung einer natürlichen Person, Gesundheitsdaten oder Daten zum Sexualleben oder der sexuellen Orientierung einer natürlichen Person." Deren Verbreitung ist untersagt (Datenschutz-Grundverordnung, Artikel 9, Absatz 1).
Was durch solche Maßnahmen nicht verhindert werden kann, ist ein allgemeiner Vertrauensverlust (16) in unsere Massenmedien als Lieferanten glaubwürdiger Informationen. Da es sich bei Vertrauen um eine weitere Grundfeste unserer Gesellschaft handelt, ist auch dieser Verlust an Vertrauen, nicht anders als der Verlust an Wahrheit, als gesellschaftsgefährdend einzustufen. Wollen, oder besser: dürfen, wir der weltweiten Werbung wirklich all dies opfern? www - Nein, danke!

\section{Literatur}

1. Anklageschrift IN THE UNITED STATES DISTRICT COURT FOR THE DISTRICT OF COLUMBIA, CRIMINAL NO. (18 U.S.C. $\$ \$ 2$, 371, 1349, 1028A) (Case 1:18-cr-00032-DLF Document 1 Filed 02/16/18); https://www.justice.gov/opa/ press-release/file/1035562/download

2. Anonymus. Digital intuition. A computer program that can outplay humans in the abstract game of Go will redefine our relationship with machines (Editorial). Nature 2016; 529: 437.

3. Anonymus. Is all publicity good? New Scientist 2018; $3167: 5$.

4. Anonymus. Abmeldung bei sozialen Medien?Daten-Skandal schreckt Deutsche ab. N-tv.de, 24.3.2018 https://www.n-tv.de/panorama/DatenSkandal-schreckt-Deutsche-ab-article20352773.html?service=print

5. Anonymus. As Facebook scandal mushrooms, Mark Zuckerberg vows to step up. The Economic Times, 22.3.2018 https://economictimes.indiatimes.com/news/international/business/

...hrooms-mark-zuckerberg-vows-to-step-up/ printarticle/63407174.cms

6. Bond RM, Fariss CJ, Jones JJ, Kramer ADI, Marlow C, Settle JE, Fowler JH. A 61-million-person experiment in social influence and political mobilization. Nature 2012; 489: 295-298.

7. Cabañas JG, Cuevas Á, Cuevas R. Facebook use of sensitive data for advertising in Europe. arXiv: 1802.05030v, 14.2.2018 https://arxiv.org/ pdf/1802.05030.pdf

8. Chen A. The real paranoia-inducing purpose of Russian hacks. The New Yorker, 27. Juli 2016 https://www.newyorker.com/news/news-desk/thereal-paranoia-inducing-purpose-of-russian-hacks

9. Covington P, Adams J, Sargin E. Deep Neural Networks for YouTube Recommendations. RecSys ' 16 September 15-19, 2016, Boston, MA, USA; DOI: http://dx.doi.org/10.1145/2959100.2959190

10. Eltagouri M. The rise of 'Putin's chef', the Russian oligarch accused of manipulating the U.S. election. The Washington Post 17.2.2018 https://www. washingtonpost.com/news/worldviews/ wp/2018/02/16/the-rise-of-putins-chef-yevgeniyprigozhin-the-russian-accused-of-manipulatingthe-u-s-election/?utm_term $=.4142 \mathrm{~b} 7$ ea1d6d

11. Europäische Union. Datenschutz-Grundverordnung http://eur-lex.europa.eu/legal-content/DE/ TXT/PDF/

?uri=CELEX:02016R0679-20160504\&from=EN) 
12. Fowler JH. A Follow-up to a 61 Million Person Experiment in Social Influence and Political Mobilization. http://www.nasonline.org/programs/ sackler-colloquia/documents/fowler.pdf

13. Kosinski M, Stillwell D, Graepel T. Private traits and attributes are predictable from digital records of human behavior. PNAS 2013; 110: 5802-5805.

14. Kosinski M, Matz SC, Gosling SD, Popov V, Stillwell D. Facebook as a Research Tool for the Socia Sciences. Opportunities, Challenges, Ethical Considerations, and Practical Guidelines. American Psychologist 2015; 70: 543-556.

15. Kramer ADI, Guillory JE, Hancock JT. Experimental evidence of massive-scale emotional contagion through social networks. PNAS 2014; 111: 8788-8790.

16. Lazer DMJ, Baum MA, Benkler Y, Berinsky AI, Greenhill KM, Menczer F, Metzger MJ, Nyhan B, Pennycook G, Rothschild D, Schudson M, Sloman SA, Sunstein CR, Thorson EA, Watts DJ, Zittrain JL. The science of fake news. Science 2018; 359: 1094-1096.

17. Lohr S. It's True: False News Spreads Faster and Wider. And Humans Are to Blame. New York Times. 8. März 2018. https://www.nytimes com/2018/03/08/technology/twitter-fake-news-research.html

18. Matz SC, Kosinski M, Nave G, Stillwell DJ. Psychological targeting as an effective approach to digital mass persuasion. PNAS 2017; 114: 12714-12719.

19. Nicas J. How YouTube Drives People to the Internet's Darkest Corners. Wall Street Journal 7.2.2018 https://www.wsj.com/articles/how-youtube- drives-viewers-to-the-internets-darkestcorners-1518020478

20. Revell T. What's not to like? The scale and scope of Facebook's huge ad machine has been revealed. New Scientist 2018; 3166: 4-5.

21. Rosenberg M Confessore N, Cadwalladr C. How Trump consultants exploited the Facebook data of Millions. The New York Times, 17.3.2018 https://www.nytimes.com/2018/03/17/us/politics/ cambridge-analytica-trump-campaign.html

22. Rosenberg M, Frenkel S. Facebook's role in data misuse sets off storms on two continents. The New York Times, 18.3.2018 https://www.nytimes. com/2018/03/18/us/cambridge-analytica-facebook-privacy-data.html

23. Rosenthal R. Media violence, antisocial behavior and the social consequences of small effects. Journal of Social Issues 1986; 42: 141-154.

24. Rosenthal R. How are we doing in soft psychology? Americal Psychjologist 1990: 775-777.

25. Silverman E. Facebook's first president, on Facebook: 'God only knows what it's doing to our children's brains. The Washington Post, 9.11.2017. https://www.washingtonpost.com/news/theswitch/wp/2017/11/09/facebooks-first-presidenton-facebook-god-only-knows-what-its-doing-toour-childrens-brains/?utm_term $=.8193$ cbc693c7

26. Spitzer M. Spuren in der Wolke. Mit Sozialverhalten kann man rechnen - aber wollen wir das? Nervenheilkunde 2013; 32: 253-256.

27. Spitzer M. Dopamin und Käsekuchen. Essen als Suchtverhalten. Nervenheilkunde 2010; 29: 482-486.
28. Spitzer M. Qatar. Eine deutsche schule, islamische Kunst und ein Land, das nachdenklich macht. Nervenheilkunde 2018; 37: 125-135.

29. Thubron R. YouTube's ,recommended videos' algorithm keeps surfacing controversial content. Despite Googles tweaks. Techspot 8.3.2018 https://www.techspot.com/news/73178-youtuberecommended-videos-algorithm-keeps-surfacingcontroversial-content.html

30. Troianovski A, Helderman RS, Nakashima E, Timberg C. The 21st-century Russian sleeper agent is a troll with an American accent. The Washington Post, 17.2.2018. https://www.washingtonpost.com/ business/technology/the-21st-century-russiansleeper-agent-is-a-troll-with-an-american-accent/ 2018/02/17/d024ead2-1404-11e8-8eal-cld91fce c3fe_story.html

31. Tufekci Z. YouTube, the great redicalizer. The New York Times 2018; 12: 15.

32. Verma IM. Editorial Expression of Concern and Correction. PNAS 2014; 111: 10779.

33. Vosoughi S, Roy D, Aral S. The spread of true and false news online. Science 2018; 359: 1146-1151.

34. Wu Y, Kosinski M, Stillwella D. Computer-based personality judgments are more accurate than those made by humans. PNAS 2015; 112: 1036-1040.

35. Meyer R. My Facebook Was Breached by Cambridge Analytica. Was Yours? How to find out if you are one of the 87 million victims. The Atlantic, 10.4.2018 https://www.theatlantic.com/technol ogy/archive/2018/04/facebook-cambridge-analytica-victims $/ 557648$ 